\title{
Monologue intérieur et éclats impressionnistes : traduction du début de « Bedsitter » d'Eva Figes (1932-2012)
}

\author{
Nathalie Vincent-Arnaud \\ Traducteur : Nathalie Vincent-Arnaud
}

\section{OpenEdition}

\section{Journals}

Édition électronique

URL : https://journals.openedition.org/ml/5294

DOI : $10.4000 / \mathrm{ml} .5294$

ISSN : 2274-0511

\section{Éditeur}

Association Modèles linguistiques

\section{Édition imprimée}

Date de publication : 30 décembre 2017

Pagination : 195-203

\section{Référence électronique}

Nathalie Vincent-Arnaud, « Monologue intérieur et éclats impressionnistes : traduction du début de « Bedsitter » d'Eva Figes (1932-2012) », Modèles linquistiques [En ligne], 76 | 2017, document 8, mis en ligne le 10 juin 2019, consulté le 01 juillet 2021. URL : http://journals.openedition.org/ml/5294 ; DOI : https://doi.org/10.4000/ml.5294 


\section{Monologue intérieur et éclats impressionnistes : traduction du début de « Bedsitter » d’Eva Figes (1932-2012)}

\section{Nathalie Vincent-Arnoux}

Si Les deux pages qui font l'objet de la traduction ci-après sont extraites de "Bedsitter ", texte d'Eva Figes publié en 1975 dans le recueil Signature Anthology, vingtième volume de la collection Signature Series. C'est Beckett, dont le très court texte «Still » ouvre ce recueil, qui est l'un des plus éminents représentants de la collection, le volume 9, paru en 1970 sous le titre éloquent de Lessness, étant en effet entièrement consacré à des écrits en prose de l'auteur irlandais. En écho à ce nom et aux résonances qu'il engendre, la notice éditoriale présentée en quatrième de couverture permet de se faire une idée assez précise des orientations stylistiques de cette collection :

The Signature Series was established to publish works by writers of the highest quality that is specifically idiosyncratic in form, length, or subject matter. Each volume bears the unmistakable signature of its author, perhaps in the sense that each represents a personal idea that could not be expressed or arranged in conventional forms.

L'association du nom de Beckett et de celui d'Eva Figes n'est pas ici une première, une telle rencontre s'étant déjà produite quelques années auparavant, en 1967, lors de la publication du deuxième roman d'Eva Figes, Winter Journey, qui avait valu à celle-ci les hommages d'une critique impressionnée au premier chef par une certaine parenté avec l'auteur de L'Innommable. « Reminiscent of Beckett...a kind of poetry ", titra ainsi le Guardian à l'époque, bien en peine d'assigner une quelconque étiquette générique à un écrit qui, comme beaucoup d'autres qui suivirent, demeure à jamais inscrit dans cette 
zone intermédiaire, règne du " phrasé-parlé » (Martin, 26), du monologue intérieur aux configurations variables, de l'incertitude référentielle, caractéristique de la plupart des productions fictionnelles d'Eva Figes. «Bedsitter »- auquel un autre court texte, « Obligato », succède en fondu enchaîné tel un fragment subitement détaché du précédent (Vincent-Arnaud, 2009) - ne fait pas exception à ce qui fait désormais office de marqueur stylistique d'une œuvre fictionnelle échelonnée sur trois décennies (de 1966 à 1996) et juxtaposée à des écrits d'ordre sociologique mais aussi autobiographique, cette dernière dimension ayant complètement éclipsé les deux précédentes dans les dernières années de la vie de l'auteur avec la parution de Tales of Innocence and Experience. An Exploration (2003) puis de Journey to Nowhere (2008).

Bien loin de constituer un changement radical de perspective, cette évolution générique d'une fiction expérimentale - favorisée par la participation d'Eva Figes, dans les années soixante, à un cercle informel de jeunes auteurs - jusqu'aux écrits autobiographiques plus tardifs est au contraire marquée par la persistance d'une veine profondément intimiste, $d^{\prime}$ une combat jamais interrompu avec le « vertige de l'innommable» (Kristeva, 103). Comme en écho à la loi du silence opiniâtre imposée par sa mère à propos d'une histoire familiale confrontée aux horreurs de l'Histoire, la venue à l'écriture d'Eva Figes s'est d'abord faite par le truchement de masques fictionnels multiples destinés à reléguer prudemment au second plan le «je » aux assises troublées de l'auteur, même si c'est invariablement, d'une œuvre à l'autre, puis d'un genre à l'autre, la même trame qui se loge dans le récit : celle qui donne voix à des êtres en proie à une errance identitaire interminable, taraudés par l'obsession d'un passé aussi diffus que lourdement perturbant. La fracture existentielle, les éclats d'une affectivité composant autant de réflecteurs des traumatismes de l'Histoire comme des fragments d'une histoire individuelle, tels sont les invariants qui sous-tendent une œuvre assimilable à « un monde [...] à l'intérieur duquel règne une loi homogène, une nécessité de type organique » (Starobinski, 34). L'organicité dont procède cette écriture « en souffrance $» 58$ prend naissance dans une voix bri-

58. J'utilise ici l'image qui donne son titre à l'ouvrage de Marc Amfreville, Écrits en souffrance, Paris, Michel Houdiard éditeur, 2009. 
sée, amenée à renaître autrement, dans un autre idiome, et portant à fleur d'écriture les stigmates de cette dislocation précoce qui a entraîné cette prolifération des figures de l'exil et de la hantise.

C'est en 1939, à l'âge de sept ans, qu'Eva Figes, née à Berlin dans une famille juive-allemande, arriva en Angleterre avec ses parents et son frère afin de fuir les persécutions du régime nazi dont son père avait déjà été victime et auxquelles ses grands-parents, déportés, allaient bientôt succomber. Outre le silence maternel obstiné qui s'ensuivit, seulement rompu après la découverte de la Shoah, bien plus tard, par l'adolescente lors d'une séance de cinéma à Londres, cette fuite précipitée engendra un exil linguistique subit dont l'auteur relate de manière répétée les épisodes les plus marquants dans ses écrits autobiographiques. Néanmoins, sur cette nouvelle identité britannique endossée à la hâte par la force des événements, la langue anglaise imprima durablement sa marque jusqu'à devenir langue d'écriture unique, louée par la critique et valant à l'auteur les comparaisons flatteuses déjà évoquées auxquelles devait s'ajouter, un peu plus tard, la reconnaissance d'une certaine filiation avec Virginia Woolf.

Certains des traits les plus patents de cette filiation identifiée par la critique s'affichent dans le court roman Light, mettant en scène Claude Monet et paru en 1983. Le titre même de ce roman, son incipit et son excipit centrés sur les variations du ciel et de la lumière, la structure temporelle d'ensemble (une seule journée parcourue de l'aube à la nuit), la présence d'un personnage focalisateur du nom de Lily (relayé par les « lilies » et autres « lily ponds » qui, allégories de l'art rédempteur, ornent le jardin du peintre), les allusions à la Première guerre mondiale et aux deuils subis par les personnages sont autant d'éléments qui, seuls ou combinés les uns aux autres, font immanquablement signe vers l'univers woolfien, de To the Lighthouse (1927) à The Waves (1931). Forme de respiration paradoxale au sein d'un univers fictionnel par ailleurs invariablement lourd et sombre où le huis-clos mental se substitue au jardin lumineux de Monet, ce roman n'en est pas moins marqué, lui aussi, par la domination du monologue intérieur et de ses formes dérivées, même si ces dernières se glissent dans une myriade de points de vue et 
d'impressions naviguant d'un personnage à l'autre.

Toutefois, loin de ne concerner que des éléments structurels, paratextuels ou onomastiques - qui n'en sont pas moins dignes d'attention -, l'ampleur de l'empreinte woolfienne se mesure surtout aux «faits de texture » (Adam, 19) qui jalonnent les itinéraires singuliers de ces voix intérieures. Dans la plupart des autres œuvres de fiction, romans ou shorter works résistant à toute catégorisation précise tel que le présent texte "Bedsitter ", c'est une conscience solitaire qui, aux prises avec son déchiffrement du monde et d'ellemême, vient occuper sur le mode autodiégétique le devant de la scène en y déposant d'emblée les marques de son exil et de son autarcie énonciative. Exemples saisissants des « singularités " ${ }^{59}$ en forme d'aspérités qui jalonnent ces œuvres où syntaxe et clarté référentielle ont tôt fait de voler en éclats, Winter Journey - qualifié de «disquieting » par la critique lors de sa parution en 1967 - et Konek Landing (1969), figurent ainsi au nombre des premiers romans pouvant être décrits comme " clear inheritors of stream-ofconsciousness techniques » (Pellicer Ortin, 72). La carcéralité des consciences ainsi représentées fait irruption, dans de nombreux passages, à travers un texte dé-chaîné, règne du fragmentaire et des " images brisées » ${ }^{60} \mathrm{~d}$ 'intériorités en mal de repères et d'ancrage stabilisateur, errant quelque part aux confins de leur mémoire hantée tel Janus, narrateur de Winter Journey:

Forget. The clock ticking over. Let go, back black, pitch black midnight, not a glimmer, soft coals on my eyes and in my skull. Nothing stirs, not a bird. Nothing is. Who wants light anyway. Let it go, let it wash, backwash, dark waves breaking, washing round my skull. Locked in chaos, the dark cave. (Figes, 1967, 8)

«Bedsitter » paraît offrir une réduplication, sous forme condensée, de ces modalités représentatives d'un huis-clos assiégé par des voix, des souvenirs, des obsessions, même si la situation qu'il évoque gagne un peu en clarté par rapport à la hantise mystérieuse parcourant l'extrait cité précédemment. La chambre meublée où se tient le

59 J'emploie ici le terme de Jean-Jacques Lecercle (2008).

60. Traduction partielle, désormais consacrée, de « heap of broken images », célèbre expression de The Wasteland de T.S. Eliot. 
monologue du personnage - à l'identité inconnue - en déroute financière et existentielle est le lieu du surgissement d'un " chaos » mental similaire, «grotte sombre » peuplée de souvenirs et de pensées obsédantes. Le titre même du texte (en anglais comme dans la traduction française proposée) suffit à évoquer, par-delà sa valeur dénotative, le caractère confiné et encombré $\mathrm{d}^{\prime}$ une conscience qui, dans sa tentative angoissée d'évaluation de ses moyens de subsistance, se fait carrefour d'impressions et de réminiscences fulgurantes.

Si l'incipit est réductible au premier paragraphe qui constitue à lui seul une entrée en matière suffisamment éloquente en raison des modalités énonciatives et des repères spatio-temporels qu'il instaure ou du moins suggère, les trois paragraphes réunis ci-après se constituent en unité modélisante de la dynamique textuelle qui se met en place. À l'hypotypose de l'errance mentale dessinée par le collage fragmentaire en forme de parataxe incessante, ellipses, répétitions, abruptions et détours subits, particules nomades non identifiées ( « they / « them »), se mêle, via l'anaphore et le connecteur présents à l'ouverture des deux derniers paragraphes, une logique de la divagation qui assure la cohérence paradoxale du discours (" This is no use, not facing up to it / « So: time is running out, always was »). Le motif centripète de la noirceur qui parcourt la fin du dernier paragraphe («black void » / «black hollow space ») fournit l'une des cellules motrices de l'ensemble d'un texte dont l'imaginaire contribue à son tour à tisser la toile de fond, dense et oppressante, « sounds in the dark » (Figes, 1967, 8), « dark space » (Figes 1989, 1) et autres « dark waves breaking » (Figes, 1967, 8), sur laquelle s'édifie régulièrement, d'une œuvre à l'autre, le discours des voix intérieures.

Comme l'a très bien conçu et formulé Monique De Mattia-Viviès dans son ouvrage sur le discours indirect libre,

[...] il n'est pas possible de remonter d'une représentation romanesque à une réalité psychique ; tout au plus la représentation romanesque nous permet-elle de voir comment, à un moment donné, une communauté culturelle pu concevoir une réalité psycho-linguistique précise. (De Mattia-Viviès, 216) 
Il en est ainsi de la représentation opérée par le monologue intérieur chez Eva Figes, même si le «moment donné » évoqué plus haut ne peut ici, de toute évidence, s'appréhender dans sa finitude, tout comme il paraît désormais sensé d'envisager l'œuvre estampillée « moderniste »- en tant que produit scriptural ou artistique relevant d'un certain mode de saisie et d'expression du réel - dans une perspective trans-historique, trans-générationnelle. ${ }^{61}$ À travers la forme «idiosyncratique » qu'elle a pratiquée critère de sélection mis en avant par la collection Signature Series (voir supra) -, Eva Figes s'est bel et bien affirmée comme héritière de plein droit d'un Modernisme dont l'émergence, d'un point de vue historique, s'enracine dans une fracture historique et existentielle analogue à celle qui a déterminé son propre parcours. Traversée de tous ces échos, sa voix s'impose à travers des textes qui sont autant de défis herméneutiques renouvelés et qui, par la force du chaos dont ils émanent, ne peuvent que résister. La traduction proposée ci-après permettra, espérons-nous, d'éprouver cette résistance.

Université Toulouse-Jean Jaurès CAS (Cultures Anglo-Saxonnes, EA 801)

\section{Chambre Meublée}

L'électricité, vingt, mais non voyons, beaucoup plus que ça en hiver, c'est quand le prochain hiver, dans neuf mois, autant dire des siècles, et le loyer qui tombe à la fin du mois, et encore vingt, bon sang, et je n'aurai bientôt plus un sou en poche, quinze par semaine qui s'en vont, faut que je m'arrête de fumer, faut que je boive moins, où estce que j'ai mis les allumettes, où j'en étais, ah oui, je disais que le plus important c'est de se concentrer sur le travail, produire, aller de l'avant, sans faire attention aux petits détails comme tous ceux qui se laissent avoir par tout ça, tous ces esprits étroits, qu'importe demain, et les journaux, faudrait que je récupère les vieux au lieu d'en acheter de nouveaux, de toute façon, à quoi bon lire toutes ces âneries, et puis, quand même, il faut que je prenne une décision, et si je vendais ces livres, et ça fait combien de temps que je n'ai pas travaillé maintenant, deux jours? Trois ? Et tout ce travail, de toute façon, à quoi

61. Ce point de vue rejoint celui qui est exprimé par Silvia Pellicer Ortin concernant Eva Figes : « My study will show that it may be hard to establish clear-cut boundaries between the Modernist and Postmodernist traits of Figes' work, and that she is better approached from an intermediate critical position that combines Modernist and Postmodernist traits » (Pellicer Ortin, 72). 
ça a servi, tous ces mois ? Faut que je prenne une décision, j'peux pas rester comme ça, sans rien faire, peut-être que je pourrais arrêter de me nourrir, me laisser dépérir, comme ça, peut-être que ça serait une solution, et après, qu'est-ce qu'ils feraient de moi, peut-être qu'ils jetteraient mon corps aux ordures avec tout un tas d'objets encombrants, peut-être que je serais tellement maigre qu'il ne resterait plus rien de moi, qu'il n'y aurait pas de réponse quand on frapperait à la porte, ça serait marrant, ça, que la vieille fille ne puisse plus venir quémander ses loyers impayés, j'voudrais bien voir la tête qu'elleferait.Pas la peine tout ça, rien à faire pour en sortir. C'est toujours la même chose, je m'assois là avec un stylo-bille et un bout de papier, pour faire les comptes, et puis à chaque fois je renonce avant même de commencer, tout ça parce qu'il n'y a pas besoin d'attendre longtemps pour voir ce mur qui se dresse, là, devant moi. Les dépenses, voyons... La nourriture, cinq, le loyer, encore cinq, le chauffage, deux, l'électricité, Dieu sait combien, les cigarettes, quatre, et puis l'électricité le papier les livres le linge et merde et les chaussures et lire et faire la conversation et enfin foutre le camp. Tout ça, ça se paie. Mais l'argent qui rentre, zéro. Le bout de papier, y a qu'à se torcher avec.Voilà, le temps s'enfuit, rien de nouveau dans tout ça, faut penser au travail, et seulement au travail, c'est ça qui compte, ne pas faire attention à quoi que ce soit d'autre, et on a seulement quelques heures, quelques années peut-être, ne pas regarder en arrière ou en bas, ne pas perdre l'équilibre, ne pas tomber, ne pas devenir fou à cause d'eux, ces enragés, tous ces enragés qui n'arrêtent pas, montrer ce qu'on a à l'intérieur, le révéler au monde ahuri, ils seront tous ahuris et stupéfaits, je vous promets, tout ça je vais le faire, c'est quoi déjà la phrase célèbre, les mots se noient dans un vide sidéral, ils se perdent dans le noir, oui, tout ça je le ferai, la voix de l'acteur, puissante, pleine de passion, ses échos qui résonnent dans le noir, rideau de scène, répétition, c'était quoi, déjà, le nom de la pièce?

Eva Figes, 'Bedsitter', Signature Anthology (Signature Series 20), London, Calder \& Boyars, 1975, p. 33-38. (traduction : Nathalie Vincent-Arnaud 


\section{Bibliographie}

ADAM, J-M. (1994), «Style et fait de style : un exemple rimbaldien », Qu'estce que le style ? (Georges Molinié et Pierre Cahné, éd.), Paris, Presses Universitaires de France, p. 15-43.

AMFREVILLE, M. (2009), Écrits en souffrance, Paris, Michel Houdiard éditeur.

DE MATTIA-VIVIES, M. (2006), Le Discours indirect libre au risque de la grammaire. Le cas de l'anglais, Aix-en-Provence, Presses de l'Université de Provence.

FIGES, E. (1975) «Bedsitter », in Signature Anthology (Signature Series 20), London, Calder \& Boyars, 1975, p. 33-38.

FIGES, E. (1967), Winter Journey, London, Panther Books.

FIGES, E. (1969), Konek Landing, London, Panther Books.

FIGES, E. (1983), Light, London, Hamish Hamilton.

FIGES, E. (1989), Ghosts, London, Flamingo.

FIGES, E. (2003), Tales of Innocence and Experience - an Exploration, London, Bloomsbury.

FIGES, E. (2008), Jouney to Nowhere, London, Granta Books.

KRISTEVA, J. (1987), Soleil noir : dépression et mélancolie, Paris, Gallimard.

LECERCLE, J-J. (2008), « Pour une stylistique des singularités », Bulletin de la Société de Stylistique Anglaise 31, Nanterre, Presses de l'Université de Paris X, p. 21-32.

MARTIN, J-P. (1998), La Bande sonore, Paris, Corti.

PELLICER ORTIN, S. (2015), Eva Figes' Writings. A Journey through Trauma, Newcastle upon Tyne, Cambridge Scholars Publishing.

STAROBINSKI, J. (1970), « Léo Spitzer et la lecture stylistique », préface à Léo Spitzer, Etudes de style, Paris, Gallimard.

VINCENT-ARNAUD, N. (2007), « 'Between two seas of sound' : de l'hypotexte musical à l'écriture poétique, les figures de la dualité dans Winter Journey (1967) d'Eva Figes », Bulletin de la Société de Stylistique Anglaise $\mathrm{n}^{\circ} 28$, Paris, Presses Universitaires de Paris X, p. 189-200.

VINCENT-ARNAUD, N. (2008), " 'Living on the edge' / "in the lost spaces" : l'écriture d'Eva Figes à l'épreuve des limites dans Tales of Innocence and Experience (2003) », Frontières, Marges et Confins, (Corinne AlexandreGarner, éd.), Paris, Presses Universitaires de Paris X, p. 151-160.

VINCENT-ARNAUD, N. (2008), « Des voix sans grammaire, ou le discours 
irrésolu : les variations du monologue intérieur dans quelques œuvres d'Eva Figes », Bulletin de la Société de Stylistique Anglaise n 30, Paris, Presses Universitaires de Paris X, p. 181-194.

VINCENT-ARNAUD, N. (2009), « L'œuvre au noir, ou comment trouver sa voix : "Bedsitter" et "Obligato" d'Eva Figes comme essais de style", Bulletin de la Société de Stylistique Anglaise n²32, Paris, Presses Universitaires de Paris X, p. 213-227.

VINCENT-ARNAUD, N. (2010), «'A secret at the heart of darkness opening up' : de Little Eden: a Child at War (1978) à Journey to Nowhere (2008), les mots de la guerre ou les batailles du silence chez Eva Figes », Miranda $\mathrm{n}^{\circ} 2$,

$<$ http://www.miranda-

ejournal.fr $/ 1 /$ miranda $/$ article.xsp?numero $=2 \&$ id article $=$ article $08-0>$. 